\title{
Approach to hepatocaval confluence during laparoscopic right hepatectomy: three variations on a theme
}

\author{
Francesca Ratti ${ }^{1} \cdot$ Federica Cipriani $^{1} \cdot$ Marco Catena $^{1} \cdot$ Michele Paganelli $^{1} \cdot$ \\ Luca Aldrighetti ${ }^{1}$
}

Received: 30 May 2016/Accepted: 31 May 2016/Published online: 20 June 2016

(C) Springer Science+Business Media New York 2016

\begin{abstract}
Introduction Due to technical challenges and reduced pool of candidates, laparoscopic major hepatectomies remain relatively limited: In particular, right hepatectomy is technically more challenging than left since it requires liver mobilization, dissection of inferior vena cava (IVC) and hepatocaval confluence (HepCC), and section of right hepatic vein (RHV).

Materials and methods Among 53 laparoscopic right hepatectomies (San Raffaele Hospital; 2013-2015), the approach to HepCC was standardized by three techniques: (1) primary approach to IVC and RHV with complete mobilization of right hemiliver; (2) anterior approach with hanging maneuver without liver mobilization (partial anterior approach-PAA); and (3) anterior approach without hanging maneuver without liver mobilization of right hemiliver (total anterior approach-TAA). The technique was defined preoperatively based on tumor size/position, IVC/RHV compression, and HepCC dislodgement. Type of parenchyma and risk of lesion rupture were also evaluated.

Results Primary approach to IVC and RHV Before liver transection and after liver mobilization, IVC dissection is performed, and RHV is isolated and suspended on a vessel loop. RHV is sectioned after parenchymal transection. Indications: no compression by tumor of IVC/RHV,
\end{abstract}

no HepCC dislodgement, soft parenchyma, no risk of lesion rupture. PAA IVC and HepCC are dissected free before transection, without previous liver mobilization; a tape is positioned in front of the anterior aspect of IVC, to perform the hanging maneuver. RHV section is performed after parenchymal transection. Indications: huge masses without compression of IVC/RHV, no HepCC dislodgement, liver stiffness, risk of lesion/parenchyma rupture. TAA Both IVC and RHV dissections are performed at the end of parenchymal transection, without previous mobilization of right lobe. Indications: huge masses with compression of IVC/RHV, HepCC dislodgement.

Conclusion Different approaches are available for HepCC dissection during laparoscopic right hepatectomy: Liver parenchyma characteristics, tumor size, and relationship with HepCC should be considered in surgical planning, to achieve satisfactory outcomes.

Keywords Laparoscopy $\cdot$ Liver surgery $\cdot$ Right hepatectomy $\cdot$ Hepatocaval confluence $\cdot$ Anterior approach

Compliance with ethical standards

Disclosure The authors of this manuscript have no conflicts of interest to disclose concerning any commercial interest that they may have in the subject of study and the source of any financial or material support.
Electronic supplementary material The online version of this article (doi:10.1007/s00464-016-5015-6) contains supplementary material, which is available to authorized users.

\section{Francesca Ratti}

ratti.francesca@hsr.it

1 Hepatobiliary Surgery Division, IRCCS San Raffaele Hospital, Via Olgettina 60, 20132 Milan, Italy 